\title{
Planar cell polarity effector gene Intu regulates cell fate-specific differentiation of keratinocytes through the primary cilia
}

\author{
D Dai ${ }^{1}$, L Li', A Huebner ${ }^{1,2}$, H Zeng ${ }^{3}$, E Guevara ${ }^{4}$, DJ Claypool ${ }^{1}$, A Liu ${ }^{3}$ and J Chen ${ }^{\star, 1,5}$
}

Genes involved in the planar cell polarity (PCP) signaling pathway are essential for a number of developmental processes in mammals, such as convergent extension and ciliogenesis. Tissue-specific PCP effector genes of the PCP signaling pathway are believed to mediate PCP signals in a tissue- and cell type-specific manner. However, how PCP signaling controls the morphogenesis of mammalian tissues remains unclear. In this study, we investigated the role of inturned(Intu), a tissue-specific PCP effector gene, during hair follicle formation in mice. Tissue-specific disruption of Intu in embryonic epidermis resulted in hair follicle morphogenesis arrest because of the failure of follicular keratinocyte to differentiate. Targeting Intu in the epidermis resulted in almost complete loss of primary cilia in epidermal and follicular keratinocytes, and a suppressed hedgehog signaling pathway. Surprisingly, the epidermal stratification and differentiation programs and barrier function were not affected. These results demonstrate that tissue-specific PCP effector genes of the PCP signaling pathway control the differentiation of keratinocytes through the primary cilia in a cell fate- and context-dependent manner, which may be critical in orchestrating the propagation and interpretation of polarity signals established by the core PCP components.

Cell Death and Differentiation (2013) 20, 130-138; doi:10.1038/cdd.2012.104; published online 31 August 2012

The planar cell polarity (PCP) or noncanonical Wnt (Wnt/PCP) signaling pathway is an evolutionarily conserved signaling mechanism, essential for a number of development events such as break of symmetry, ${ }^{1-5}$ convergent extension, ${ }^{6}$ ciliogenesis, ${ }^{7}$ and polarization of stereocilia and kinocilia of inner ear epithelial cells of the cochlea. ${ }^{8}$ Currently, it is believed that core PCP proteins, such as Frizzled 6 (Fzd6), Van Gogh-like 1 and 2 (Vangl1 and Vangl2), Dishevelled 2 and 3 (Dvl2 and Dv/3), and Celsr1 are required for the establishment and propagation of planar cell polarity signals, ${ }^{9,10}$ whereas PCP effectors, such as Inturned (Intu), Fuzzy (Fuz), and Fritz (Wdpcp) of the tissue-specific PCP effector group, act downstream of the core PCP components to mediate PCP signals in a tissue- and cell type-specific manner. ${ }^{7,10}$ The genetic network and the molecular mechanisms through which PCP controls the morphogenesis of mammalian tissues remain unclear.

Disruption of core PCP genes can result in convergent extension abnormalities, such as spina bifida, ${ }^{11-14}$ whereas the disruption of tissue-specific PCP effector genes results in pleiotropic phenotypes related to abnormal formation or function of the primary cilia, such as polydactyly and exencephaly. ${ }^{15-18}$ These distinctive sets of phenotypes associated with the loss of core PCP and tissue-specific PCP effector genes suggest that these two groups of PCP genes may participate in tissue morphogenesis in a tissuespecific and context-dependent manner. To precisely determine the molecular mechanisms through which various PCP components control the mammalian development, it is desirable to investigate an organ in which both core PCP genes and tissue-specific PCP effector genes exert welldefined functions.

The epidermis of the skin is a stratified epithelial tissue developed from the single-layered ectoderm. Fully developed epidermis undergoes terminal differentiation and cornification, whereas its homeostasis is maintained by epidermal keratinocyte stem cells that reside in the basal epidermis and the hair follicles. ${ }^{19}$ Core PCP and PCP effector genes have been associated with the development, maintenance, malignant transformation, and wound healing of the skin. ${ }^{20-24}$ In addition, PCP genes also control the orientation, differentiation, and cycling of the hair follicles, the multicellular

\footnotetext{
${ }^{1}$ Department of Dermatology and Center for Regenerative Medicine and Stem Cell Biology, University of Colorado Anschutz Medical Campus, Aurora, CO, USA; ${ }^{2}$ Graduate program in Cell, Stem Cell and Developmental Biology, University of Colorado Anschutz Medical Campus, Aurora, CO, USA; ${ }^{3}$ Department of Biology, Pennsylvania State University, University Park, PA, USA and ${ }^{4}$ Department of Arts and Science, University of Colorado Denver, Denver, CO, USA

${ }^{*}$ Corresponding author: J Chen, Department of Dermatology and Center for Regenerative Medicine and Stem Cell Biology, University of Colorado Anschutz Medical Campus, 12800 East 19th Avenue, RC-1 North, P18-8126, Mail Stop 8320, Aurora, CO 80045, USA. Tel: +303 7243050 ; Fax: +303 724 3051 ; E-mail: jiang.chen@ucdenver.edu or jiang.chen@stonybrookmedicine.edu

${ }^{5}$ Current address: Departments of Pathology and Dermatology, Stony Brook University School of Medicine, Stony Brook, NY, USA.

Keywords: Planar cell polarity; Intu; cilia; keratinocyte; epidermis; hair follicle

Abbreviations: PCP, planar cell polarity; Intu, inturned; Fuz, fuzzy, Krt14, keratin 14; Krt10, keratin 10; Krt1, keratin 1; Lor, loricrin; Flg, filaggrin; Tubg, $\gamma$-tubulin; Arl13b, ADP-ribosylation factor-like 13B; GAPDH, glyceraldehyde 3-phosphate dehydrogenase; Cre, cre-recombinase; EDTA, ethylenediaminetetraacetic acid; BrdU, bromodeoxyuridine; PBS, phosphate-buffered saline; qRT-PCR, quantitative reverse-transcribed PCR; TUNEL, terminal deoxynucleotidyl transferase-mediated deoxyuridine triphosphate nick-end labeling

Received 02.2.12; revised 16.7.12; accepted 20.7.12; Edited by RA Knight; published online 31.8.12
} 
appendages of the skin. ${ }^{25-31}$ Extensive involvements of PCP genes in skin formation and homeostasis make the skin an ideal epithelial model system to dissect the PCP signaling pathway in mammals.

The formation of hair follicles in mice relies on reciprocal interactions between the epidermal cells and the underlying dermal papilla cells that involves a number of signaling pathways common to the morphogenesis of mammalian tissues and organs. ${ }^{32,33}$ The induction of the hair follicle occurs at approximately embryonic day 14.5 (E14.5) in mice with the formation of the hair placode and hair germ, a process requiring the activation of the canonical Wnt signaling pathway. Thereafter, the hair germ elongates and invaginates into the dermis to form the hair peg, which will ultimately differentiate to become a mature hair follicle, a process requiring the activation of the Hedgehog $(\mathrm{Hh})$ signaling pathway. ${ }^{33}$ Disruption of $\mathrm{Wnt} / \beta$-catenin signaling pathway results in failure of hair germ induction, whereas its upregulation results in precocious hair follicle formation and pilomatricoma. ${ }^{34-37}$ In contrast, disruption of the $\mathrm{Hh}$ signaling pathway prohibits hair follicle differentiation, ${ }^{38-40}$ whereas its unattenuated activation results in the formation of basal cell carcinoma. ${ }^{41}$ In addition, proper formation and maintenance of the skin and hair follicles require well-orchestrated interplay among signaling pathways within the epidermal compartment and between the epidermis and dermis. ${ }^{37,42-44}$ Thus, balancing the signaling output, such as Wnt and $\mathrm{Hh}$, is critical.

$\mathrm{Hh}$ signaling requires the proper formation and function of the primary cilia. ${ }^{45,46}$ Recent studies demonstrated that the formation of the primary cilia in epidermal keratinocytes and dermal papilla cells is cell autonomous, whereas the crosstalk between epidermal/dermal cells during hair follicle development requires the primary cilia in both compartments. ${ }^{29,47}$ Recent characterization of primary cilia in epidermal differentiation and wound healing ${ }^{48,49}$ and in the development of basal cell carcinoma ${ }^{50}$ demonstrated their critical roles in the development and homeostasis of the skin.

In this study, we investigated the function of Intu, a tissuespecific PCP effector gene, in the context of skin and hair follicle development. Intu is expressed in both epidermal and dermal cells of the skin. Epidermal-specific disruption of Intu resulted in hair follicle morphogenesis arrest because of follicular keratinocyte differentiation failure. Despite the fact that the stratification and differentiation of the interfollicular epidermis was essentially indistinguishable from the controls, we demonstrate that Intu regulates the differentiation of the hair follicle through its critical role in cilia formation and $\mathrm{Hh}$ signaling, and that cilia-mediated crosstalk between epidermal keratinocytes and dermal papilla cells is essential during this process. Our findings suggested a cilia-dependent and follicular keratinocyte-specific role of tissue-specific PCP effector genes on keratinocyte differentiation, which is dispensable during the stratification and differentiation of interfollicular epidermal keratinocytes.

\section{Results}

Expression profile of Intu and epidermal-specific gene targeting. Using quantitative reverse-transcribed PCR (qRT-PCR), we determined that Intu is expressed in the skin
(Supplementary Figure S1a and b). In addition, Intu is not only expressed in the epidermal cells, but also in the dermal cells.

Loss-of-function mutant embryos of Intu do not survive beyond E15.5, a critical stage of skin and hair follicle development, because of severe developmental abnormalities, ${ }^{18}$ preventing us from studying its function in the skin. To circumvent this problem, we crossed the floxed alleles of Intu $($ IntuloxP/loxP $)$ with a transgenic mouse line expressing Crerecombinase (Cre) under the control of keratin 14 (Krt14) promoter (Tg(KRT14-cre)1Amc/J, ${ }^{51}$ and thereafter referred as $\mathrm{Krt14-Cre)} \mathrm{to} \mathrm{target} \mathrm{the} \mathrm{disruption} \mathrm{of} \mathrm{Intu} \mathrm{to} \mathrm{the} \mathrm{epidermis}$ (IntuloxP/loxP $;$ Krt14-Cre). The qRT-PCR confirmed that Intu transcript was almost completely absent in the epidermis of these mice (Supplementary Figure S1c).

Intu is not required for epidermal stratification, differentiation and barrior formation, and core PCP protein polarization. To determine whether Intu is required for skin formation, we examined epidermal stratification and differentiation of the dorsal skin of germline Intu mutants $\left(I n t u^{-/-}\right)$. At E14.5, when the stratified epidermis starts to terminally differentiate, the skins of controls and mutants $\left(I n t u^{-/-}\right)$were essentially indistinguishable (Supplementary Figure S1d). At the molecular level, there was no difference in the expression of p63 and Krt14 (Supplementary Figure S1e), suggesting that Intu is not required for the stratification of the epidermis. An examination of Krt1 revealed that the Krt1-positive cells were present normally at the suprabasal layer of $\mathrm{Intu}^{-/-}$skin (Supplementary Figure S1f), demonstrating the initiation of epidermal differentiation. Labeling of Ki67 and phospho-histone H3-positive cells revealed comparable proliferation and mitotic indexes of the control and $\mathrm{Intu}^{-/-}$skin (Supplementary Figures S1g and h). The above evidence strongly suggests that Intu is dispensable for the normal stratification, differentiation, and proliferation of early embryonic epidermis.

By E14.5, PCP has already been established in the mouse skin. ${ }^{26}$ Examination of Vangl1, a core PCP protein, revealed that it was properly polarized at the lateral membrane of basal keratinocytes of Intu ${ }^{-/}$skin (Supplementary Figure S2).

To follow skin development, we examined the dorsal skin of postnatal day 2 (P2) Intu ${ }^{\text {loxP/loxP }} ;$ Krt14-Cre mutants. Epidermis-specific disruption of Intu allowed the mutant embryos to survive to term; however, mutant pups displayed growth retardation, and none of them could survive beyond P6 (Supplementary Figure S3a). Histologically, there was no apparent skin abnormalities, except for the hair follicles (Figure 1a, Supplementary Figure S3b, and also see below). At ultrastructural level, the mutant skin displayed normal spinous and granulous cell layers, normal architectures of keratin fibrils, desmosomes, and normal distribution of keratohyalin granules (Supplementary Figure S3d).

In all skin samples examined, early and late differentiation markers, such as Krt10 and loricrin (Lor), were normally expressed in control and mutant skins (Figure 1d). qRT-PCR revealed normal expression levels of $K r t 14, K r t 10, L o r$, and Flg (filaggrin) in mutant skins (Figure 1e). These results demonstrated a normal epidermal differentiation program of the mutant skin. Mutant skin showed an increased but 
b

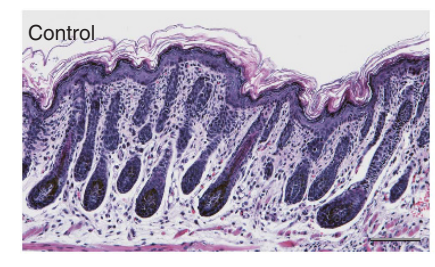

Intu ${ }^{\text {loxP/loxP }} ;$ Krt14-Cre

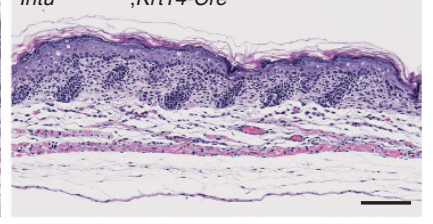

d
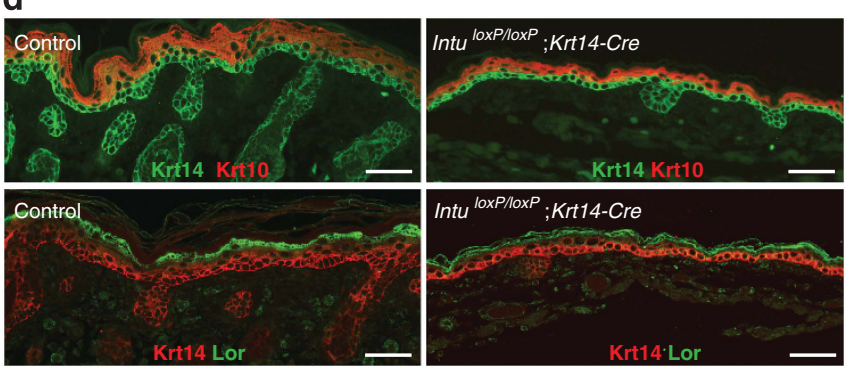

f

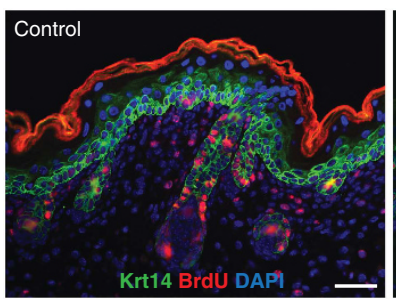

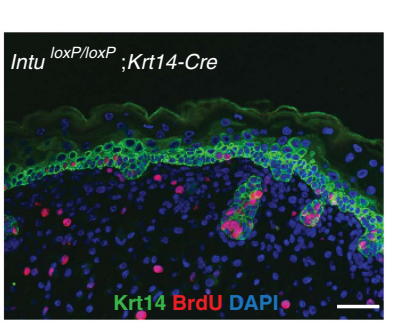

e g

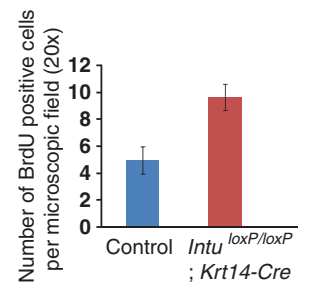

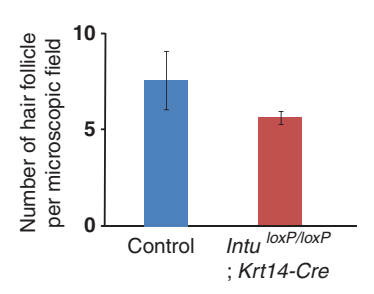

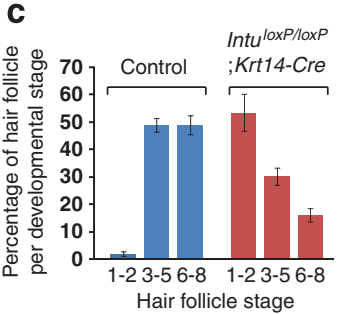

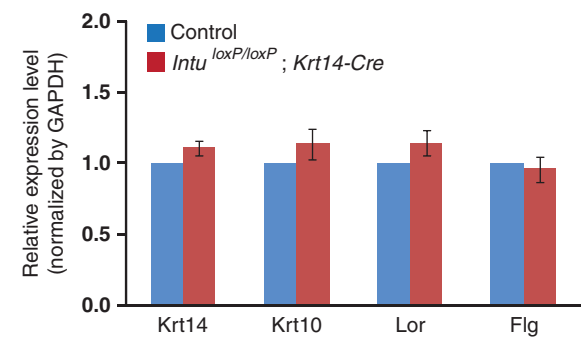

h

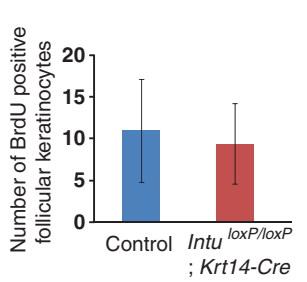

i

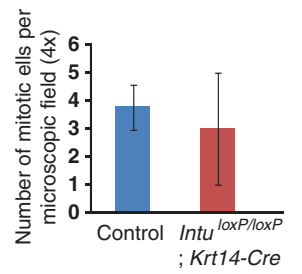

Figure 1 Hair follicle development was delayed in the dorsal skins of IntuloxP/loxP $; K r t 14-C r e$ mutants. (a-c) Histology of dorsal skin of P2 pups of Control and IntuloxP/loxP; Krt14-Cre mutants. The number of hair follicles in the mutant skin was slightly reduced (5.7 per microscopic field versus 7.6 in Control) but not statistically significant $(n=3, P=0.1904)$ (b). In comparison, the development of the hair follicles was delayed (c). Control skin contained $2 \%$ stage $1-2$ hair follicles and $49 \%$ stage $6-8$ hair follicles, whereas the mutant skin contained $53.6 \%$ stage 1-2 hair follicles and $16.1 \%$ stage $6-8$ hair follicles. (d) Expression of early and late differentiation markers of keratin 10 (Krt10; red) and loricrin (Lor; green) in the dorsal skins of E18.5 Control and Intu ${ }^{\text {loxP/loxP } ; K r t 14-C r e ~ e m b r y o s, ~ r e s p e c t i v e l y . ~ K r t 14 ~ w a s ~ l a b e l e d ~ g r e e n ~ a n d ~ r e d ~ i n ~ t h e ~ u p p e r ~ a n d ~}$ lower panels, respectively. (e) Quantitative RT-PCR demonstrated comparable expression levels of keratin 14 (Krt14), Krt10, Lor, and filaggrin (Flg) in the skins of E18.5 Control and Intu mutants (IntuloxP/loxP; Krt14-Cre). (f-h) Proliferating cells in E18.5 Control and IntuloxP/loxP; Krt14-Cre skins were labeled by BrdU (red). Krt14 was labeled green; nuclei were labeled by DAPI (blue). There was a statistically insignificant $(n=3, P=0.1399)$ increase in proliferating cells in the interfollicular epidermis of Intul ${ }^{\text {loxP/loxP }}$; Krt14-Cre mutants $(\mathbf{g})$, but comparable numbers of proliferating cells in stage 3-5 hair follicles (h). (i) Phospho-histone $\mathrm{H} 3$ labeling demonstrated similar numbers of mitotic cells in E18.5 Control and mutant skin (IntuloxP/loxP; Krt14-Cre). Scale bar: (a) $200 \mu \mathrm{m}$ and (d and f) $50 \mu \mathrm{m}$

statistically insignificant proliferation of intrafollicular epidermis (Figure 1f and $\mathrm{g}$ ), but normal expression of p63 (Supplementary Figure S4a), nuclear localization of Lef1 (Supplementary Figure S4b), apoptosis (Supplementary Figure S4c), and mitosis (Figure 1i) when compared with the control littermates. Furthermore, dye penetration assay demonstrated a normal skin barrier of Intu mutants (Supplementary Figure S3c). This characterization excluded the likelihood that the death of these mutant pups were related to skin abnormalities. Taken together, the above observations suggest that Intu is dispensable during the development of interfollicular epidermis.

To allow the mutant skin to further mature, we transplanted the dorsal skins of E18.5 control and mutant embryos onto the backs of immune-compromised $\left(\right.$ Foxn $\left.1^{-/-}\right)$recipient mice. The mutant skin was able to engraft and further develop (Figure 2a). By the fifth week after grafting, skin grafts were harvested for analysis. Histologically, the mutant grafts were able to maintain a relatively normal architecture (Figures 2a and b). Immunofluorescence labeling of Krt1 and Lor demonstrated that there was no apparent difference in epidermal differentiation between control and mutant grafts (Figure $2 b$ ). Interestingly, the epidermis of the mutant skin graft appeared hyperproliferative (Figures 2a and b), similar to the postnatal skin phenotypes observed in Hh mutants. ${ }^{38-40}$ Ki67 staining demonstrated a significant increase of proliferating epidermal keratinocytes in the mutant skin graft (Figure 2c). The expression of p63 was also increased (Figure 2d). However, apoptosis in the skin was unremarkable (Supplementary Figure S5). These observations indicate a role of Intu in the maintenance of adult epidermis.

In contrast, there is a striking difference in the number of hairs formed between the control and mutant skin grafts, the latter of which was almost completely devoid of visible hair fibers (Figure 2a, and also see below).

Intu is essential for hair follicle differentiation but not induction. Characterization of Intu ${ }^{\text {IoxP/loxP }} ;$ Krt14-Cre skin 
a
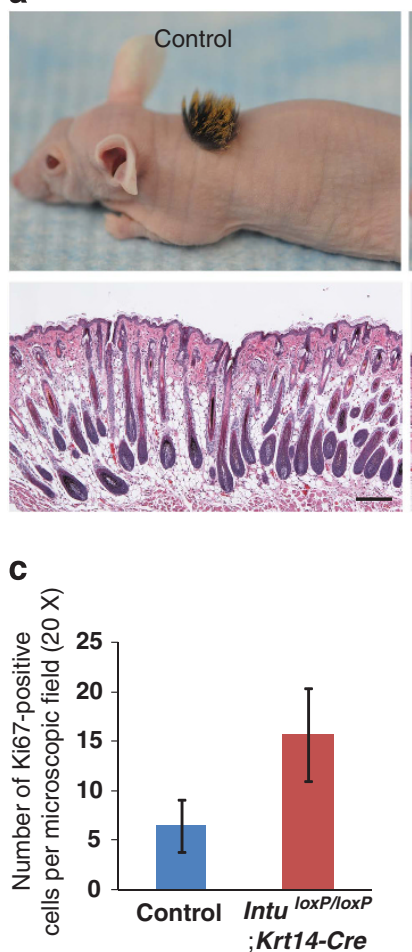

b
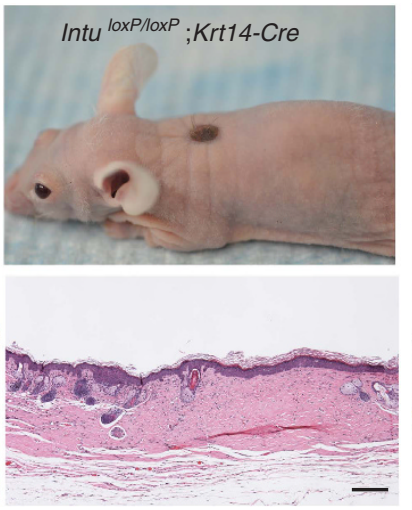
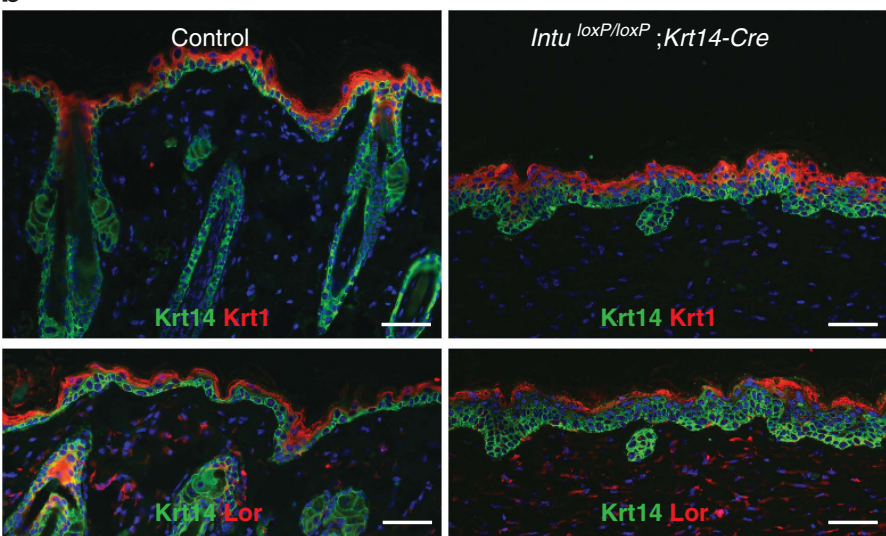

d

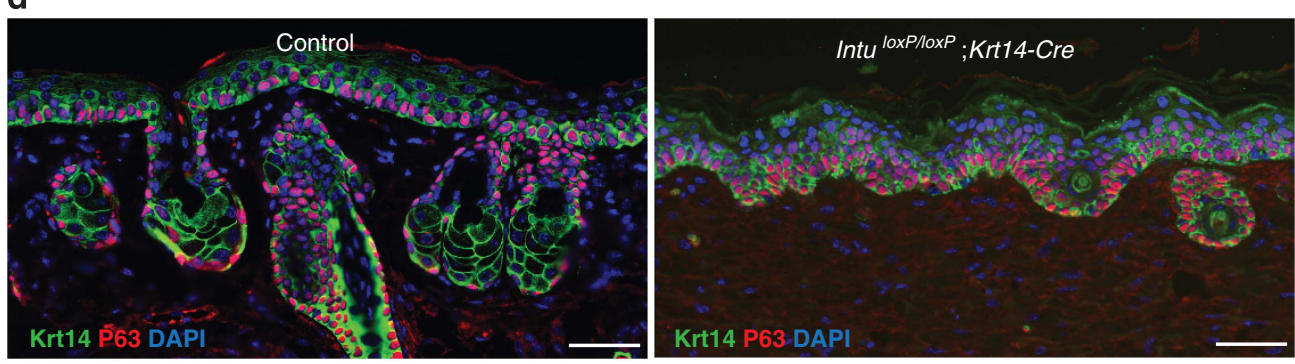

Figure 2 Hair follicles failed to grow in skin transplants of IntuloxP/loxP; Krt14-Cre mutants. (a) Gross (upper panels) and histological (lower panels) features of skin transplants from Control and IntuloxP/loxP; Krt14-Cre embryos. Note that both skin transplants survived grafting and were able to form skin. The mutant skin transplant was devoid of hair and normal hair follicles. (b) Expression of early and late differentiation marker of Krt1 (red, upper panels) and Lor (red, lower panels). (c) Ki67-positive proliferating cells in the interfollicular epidermis of IntuloxP/loxP $;$ Krt14-Cre mutants were significantly increased $(n=3, P=0.0033)$. (d) The p63-positive cells (red) in E18.5 Control and IntuloxPIloxP; Krt14-Cre skin transplants. Note that the epidermis of the IntuloxP/loxP; Krt14-Cre skin transplant was hyperproliferative with increased layers of Krt14and p63-positive cells. Krt14 was labeled green; nuclei were stained by DAPI (blue) in (b and d). Scale bar: (a) $200 \mu \mathrm{m}$ and (b and d) $50 \mu \mathrm{m}$

a

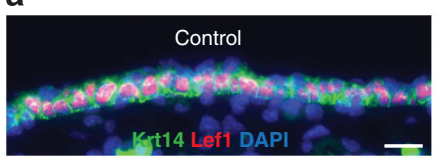

b

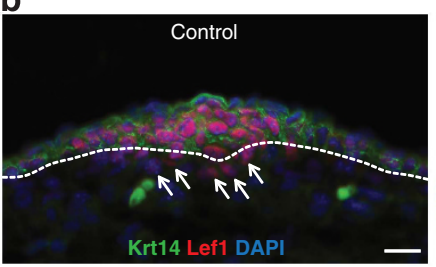

C

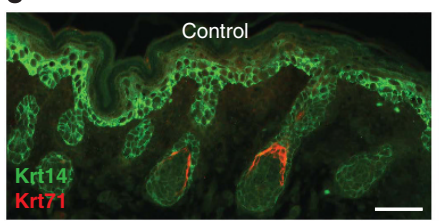

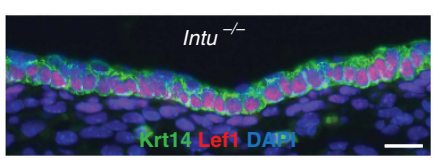
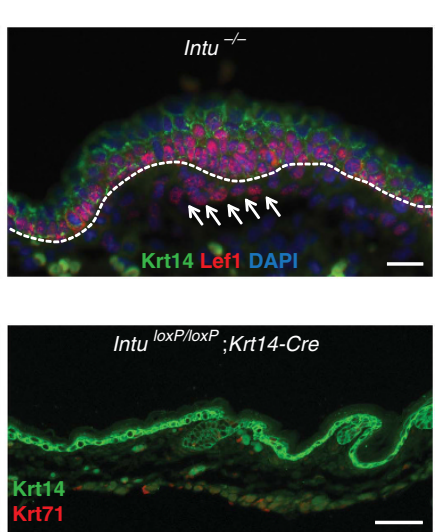

d

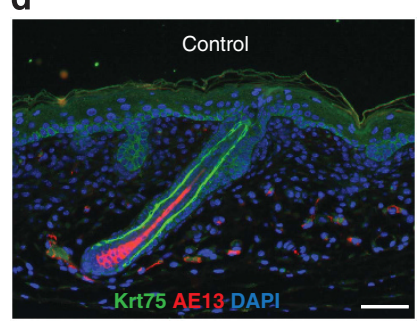

e

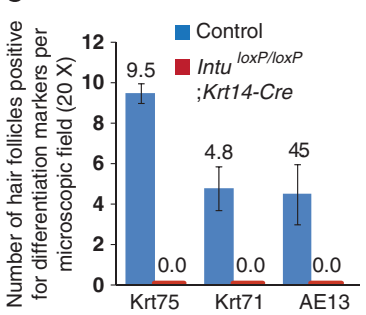

f

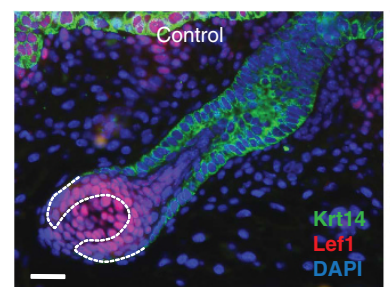

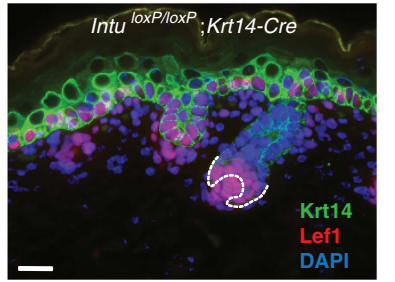

Figure 3 Hair follicle induction and differentiation in Intu mutants. (a) Nuclear expression of Lef1 (red) in the basal keratinocytes of E14.5 Control and Intu ${ }^{-/-}$skins. (b) Expression of Lef1 (red) in hair germ keratinocytes and underlying dermal cells (arrows) of Control and Intu ${ }^{-/-}$skins. Dotted line indicates the basement membrane.

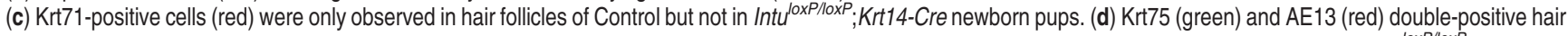
follicles were detectable in newborn Control skins. (e) Numbers of hair follicles positive for Krt75, Krt71, and AE13 in newborn skins of Control and in IntuloxP/loxP; Krt14-Cre. Note that there was no mutant hair follicle positive for any of these hair follicle differentiation markers. (f) Lef1 (red) positive cells in newborn hair follicles of Control and in Intu ${ }^{\text {IoxP/loxP }} ;$ Krt14-Cre pups. Dotted line illustrates the keratinocyte/dermal papilla boundary. Krt14 was labeled green (a-c and f); nuclei were labeled by DAPI (blue; a, b, d and f). Scale bar: (a, b, and f) $20 \mu \mathrm{m}$ and (c and d) $50 \mu \mathrm{m}$ 

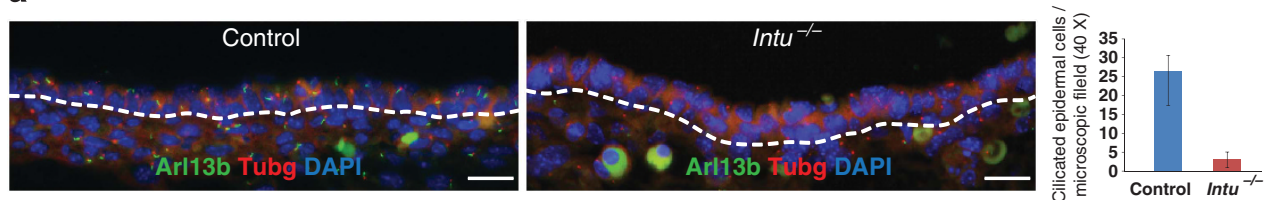

b
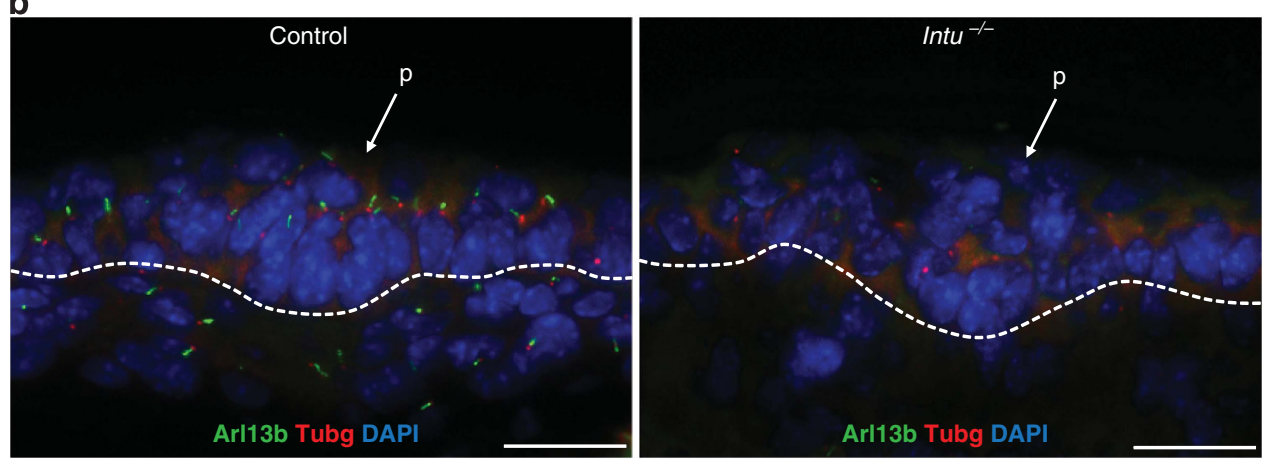

Figure 4 Primary cilia in dorsal skin of E14.5 Intu ${ }^{-1-}$ mutants. (a) Primary cilia were detected by Arl13b (green) in the epidermal keratinocytes and dermal fibroblasts of Control skins (26.4 \pm 6.6 ciliated keratinocytes per microscopic field in Control; $3.0 \pm 2.1$ in Intu ${ }^{-1-}$ mutants); $n=4, P=4.8960 \times 10^{-8}$. (b) Keratinocytes of the hair placode (p) were ciliated in Control skin but not in Intu ${ }^{-1-}$ skin. Basal bodies (red) were labeled with $\gamma$-tubulin (Tubg); nuclei were stained by DAPI (blue). Dotted line indicates the basement membrane. (a and $\mathbf{b})$ Scale bar, $20 \mu \mathrm{m}$

revealed a slight but statistically insignificant reduction of hair follicle (Figure $1 \mathrm{~b}$ and Supplementary Figures S4a and b). Based on morphological features developed by Paus et al., ${ }^{52}$ we observed a marked delay in the development of mutant hair follicles (Figure 1c). Specifically, $49 \pm 2.4 \%$ hair follicles of control skin were developed beyond stage 6 , with a small proportion $(2 \pm 0.9 \%)$ in stage 2 by $P 2$; the majority $(53.6 \pm 6.7 \%)$ of the mutant counterparts were at stages $1-2$, with only $16.1 \pm 2.5 \%$ developed beyond stage 6 (Figure 1c).

To determine whether Intu plays a permissible role during hair follicle morphogenesis, we examined hair follicles in the skin grafts. Histologically, the mutant skin grafts were almost completely devoid of hair follicles, whereas the control skin possessed an abundant number of anagen hair follicles, reminiscent of the intact skin of a normal mouse at the same age (5 weeks) (Figure $2 \mathrm{a}$, lower panels). The above observation demonstrated that Intu is essential for hair follicle development; the disruption of Intu can result in complete developmental arrest of the hair follicles.

To understand the cellular and molecular processes through which Intu controls hair follicle development, we examined key events in follicular morphogenesis. The induction of hair follicles is initiated at approximately E14.5 with the concurrent formation of the placode, aggregation of dermal condensate, and the activation of the canonical Wnt signaling. In the dorsal skin of E14.5 Intu $^{-/-}$mutants, hair placodes were able to form (Supplementary Figure S1d). Basal and follicular keratinocytes displayed an active canonical Wnt signaling as demonstrated by nuclear staining of Lef1 (Figures 3a and b). Dermal condensates were properly formed on the opposing side of these placodes and expressed nuclear Lef1 (Figure 3b). The above evidence suggests that Intu is not involved in hair follicle induction or canonical Wnt signaling in embryonic epidermis.
Because the transition to stage 6 hair follicles requires cytodifferentiation of follicular keratinocytes, we examined the differentiation of follicular keratinocytes of newborn skin by labeling of inner root sheath (IRS) cells with Krt71. As expected, a proportion of control hair follicles expressed this hair follicle differentiation marker, whereas no hair follicle in the mutant skin contained Krt71-positive cells (Figures $3 \mathrm{c}$ and e). This result was further confirmed by examining markers for the champion layer (Krt75) and hair cortex (AE13) (Figures 3d and e). Comparable numbers of proliferating cells (Figure 1h) and Lef1-positive cells (Figure 3f) in stage 3-5 hair follicles revealed no evidence to suggest that the hair follicle differentiation was prohibited by follicular keratinocytes' capability to proliferate in the Intu mutants. Furthermore, the aggregation of dermal papilla cells was unaffected in the mutants (Supplementary Figure S4d). In summary, the above evidence suggests that the hair follicle differentiation phenotypes of Intu mutants were associated with follicular keratinocytes' inability to differentiate.

Intu is essential for the formation of primary cilia and the transduction of $\mathrm{Hh}$ signals. To define the mechanism through which Intu regulates the differentiation of follicular keratinocytes, we examined the formation of the primary cilium, a microtubule-based cellular protrusion. The primary cilia are essential for processing the Hh signals, a signaling pathway essential for hair follicle differentiation. ${ }^{38-40}$ Immunofluorescence labeling revealed that cells of $I n t u^{-/-}$skin were almost completely devoid of detectable ciliary axoneme, whereas the formation and polarization of the basal body were comparable to the controls (Figure 4a). Hair placodes were able to form in Intu ${ }^{-/}$- skin; however, their cells were not ciliated (Figure 4b). Similarly, we failed to detect any normal cilia in epidermal and follicular keratinocytes in the skin of E18.5 Intu ${ }^{\text {loxP/loxP }} ;$ Krt14-Cre mutants (Figure 5a), 
a

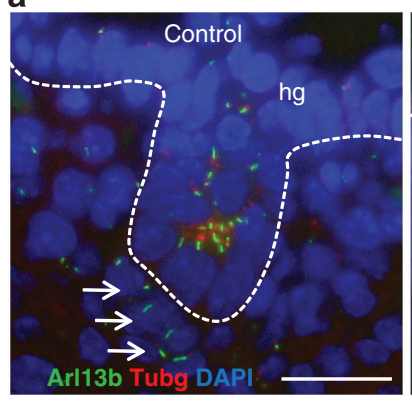

b

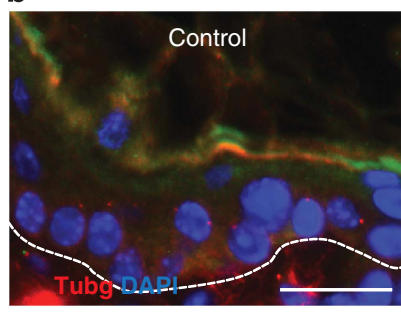

d

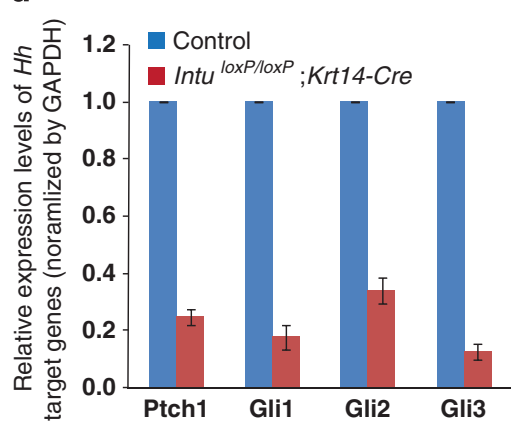

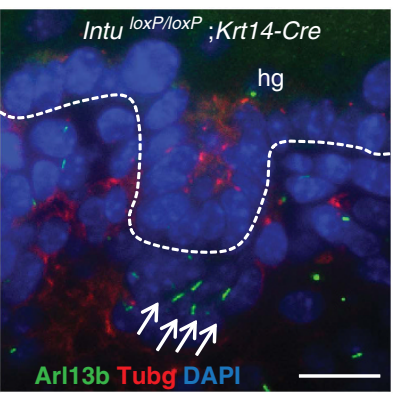

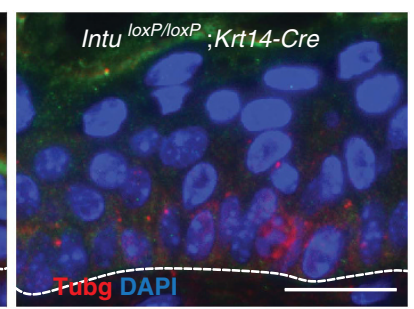

C

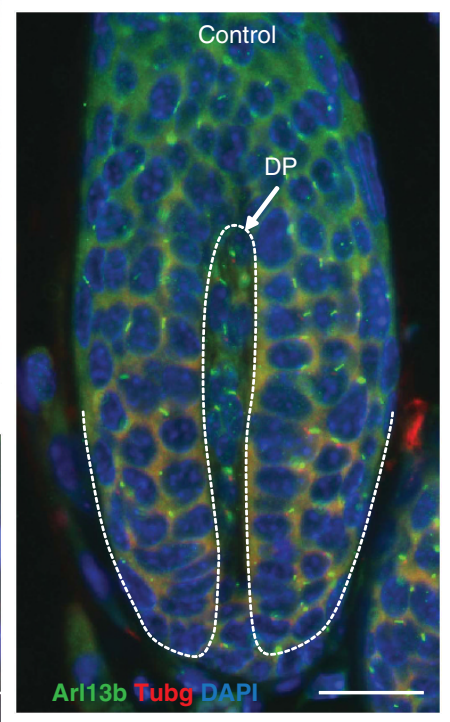

e

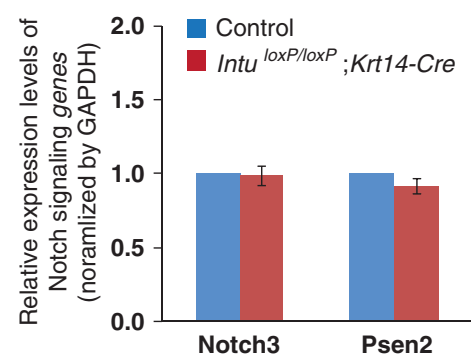

Figure 5 Primary cilia in dorsal skins of E18.5 IntuloxP/loxP; Krt14-Cre mutants and hair bulb of skin transplants. (a) Primary cilia (green) exist in hair germ (hg) keratinocytes and dermal papilla cells (arrows) of Control skins. In the skin of IntuloxP/loxP; Krt14-Cre mutants, only dermal papilla cells (arrows) were ciliated. (b) Epidermal keratinocytes of Control and IntuloxPloxP; Krt14-Cre skin transplants (equivalent to 5-week-old skin) did not contain detectable cilia. (c) Follicular keratinocytes and dermal papilla (DP) cells of a hair bulb in the Control skin transplant were ciliated. Cilia (green) were label with Arl13B, basal bodies (red) were labeled with $\gamma$-tubulin (Tubg), and nuclei were stained with DAPI (blue). Dotted line highlights the basement membrane in (a and $\mathbf{b})$ or the boundary of keratinocytes and dermal papilla cells in (c). (a-c) Scale bar, $20 \mu \mathrm{m}$. (d and $\mathbf{e})$ Quantitative RT-PCR examination of the expression of genes involved in hedgehog (d) and Notch (e) signaling pathways in skins of E18.5 embryos of Control and IntuloxP/

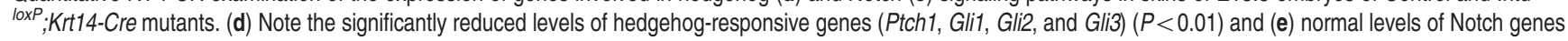
(Notch3 and Presinilin 2 (Psen2))

whereas the dermal papilla cells of the IntuloxP/loxP $; K r t 14-C r e$ skin were properly ciliated (Figure 5a). These results not only confirmed epidermis-specific gene targeting by Krt14-Cre, but also demonstrated that the hair follicle differentiation abnormality was associated with ciliogenic defects in follicular keratinocytes.

Skin grafts of controls and IntuloxP/loxP $; K r t 14-C r e$ mutants did not possess cilia as in the normal adult skin (Figure 5b). Because only control grafts contained hair follicles, we examined the hair follicle bulb and confirmed that both follicular and dermal papilla cells were ciliated (Figure 5c).

Subsequently, we examined $\mathrm{Hh}$ signaling in the mutant skin. qRT-PCR demonstrated a suppressed Hh signaling pathway (Figure 5d), confirming that the inability of the mutant hair follicles to differentiate was due to the lack of Hh signaling secondary to the loss of primary cilia. Interestingly, expression levels of genes involved in Notch signaling were unaffected (Figure 5e).

\section{Discussion}

In this study, we demonstrated that Intu, a tissue-specific PCP effector gene, plays an indispensable role in the differentiation of follicular but not epidermal keratinocytes, thus providing an example of a cell fate- and contextdependent function of PCP genes during mammalian development.

Core PCP genes of the PCP signaling pathway are involved in polarizing hair follicles along the major body axes. ${ }^{25-27}$ Tissue-specific PCP effector genes function downstream of the core PCP genes to mediate and propagate the PCP signals. During the characterization of the Intu mutants, we only observed hair follicle differentiation abnormalities, whereas polarization of core PCP proteins (Vang/1) and orientation of hair follicles of the mutants were normal. This observation confers with the concept that PCP effectors function downstream of core PCP components. 
Developing hair follicles establish their planar orientation early at hair germ stage, which is well before hair follicles start to differentiate. Not only are tissue-specific PCP effectors essential for hair follicle differentiation (this study and Dai et al. ${ }^{29}$ ), PCP effector genes of small GTPases, such as Rac1 and $\mathrm{Cdc42}$, have also been implicated in hair follicle differentiation and maintenance. ${ }^{30,31}$ Thus, the segregating hair follicle phenotypes between core PCP and PCP effector mutants also suggest that these two groups of PCP genes may control different molecular and cellular mechanisms, at least, during hair follicle development.

Intu is required for primary cilia formation in a wide range of mammalian cell types. ${ }^{18,53,54}$ Recent studies demonstrated that primary cilia are critical for the development and homeostasis of the epidermis through the regulation of p63 and Notch signaling. ${ }^{48,55}$ In this study, we utilized three in vivo model systems (a germline mutant model, a tissuespecific mutant model, and a skin grafting model) to disrupt the expression of Intu throughout the development of the skin. In the absence of Intu, primary cilia formation was disrupted and hair follicle formation arrested. Yet, despite the lack of cilia, we did not observe apparent epidermal defects, including epidermal stratification, proliferation, differentiation, mitosis, apoptosis, and barrier function, but observed normal expression levels of genes involved in epidermal differentiation and Notch signaling in embryonic skin. The drastically different phenotypes suggest that ciliogenic genes, such as Ift88 and Kif3a, and tissue-specific PCP effector genes, such as Intu, Fuz, and Wdpcp, may control cilia formation and function through different mechanisms, and further warrant the strictly context-dependent functions of tissue-specific PCP effector genes during epidermal and hair follicle differentiation. It is possible that these ciliogenic genes may perform additional cellular functions in keratinocytes beyond controlling primary cilia formation. Further investigation is required to identify novel functions of these genes (tissue-specific PCP effector genes and ciliogenic genes) beyond participating in primary cilia formation.

Canonical Wnt/ $\beta$-catenin and $\mathrm{Hh}$ signals are two of the most important signals required for hair follicle induction and differentiation, respectively. ${ }^{56}$ The primary cilium is a signaling center, critical for both signaling pathways. ${ }^{45,46}$ Disrupting Intu almost completely disrupted primary cilia and resulted in a hair follicle phenotype reminiscent of that of $\mathrm{Hh}$ mutants. ${ }^{38-40}$ However, neither hair follicle induction nor the canonical Wnt signaling pathway was affected. This evidence suggests that the primary cilium is essential for Hh signaling pathway but not canonical Wnt/ $\beta$-catenin signaling during the embryonic development of the epidermis.

The morphogenesis of hair follicles requires extensive reciprocal interactions between the keratinocytes and dermal fibroblasts. Epidermal-specific disruption of Intu prohibited the differentiation of hair follicles without affecting ciliogenesis in dermal papilla cells. This study provided further evidence that the formation of primary cilia in epidermal and dermal cells is cell autonomous, whereas the primary cilia are required for the crosstalks between follicular keratinocytes and dermal papilla cells during hair follicle differentiation, a correlation remarkably similar to previous reports. ${ }^{29,47}$ Because of the fully penetrant hair follicle differentiation and ciliogenesis phenotypes in the Intu and Fuz mutants, our studies suggest that these PCP effectors do not play redundant roles during skin development. Recent studies on Xenopus epidermis strongly suggest that these tissue-specific PCP effectors control ciliogenesis through different molecular mechanisms. ${ }^{16,17,53}$ The exact molecular function and genetic interaction of this group of PCP effector genes remain to be elucidated in mammals, and, as suggested by this study, in a cell type- and cell fate-specific manner.

The activity of Krt14-Cre in the dorsal skin starts at approximately E14.5 concomitant with hair follicle induction. ${ }^{51}$ The combined investigation of germline mutants $\left(I n t u^{-/}\right)$at E14.5 and tissue-specific knockout mutants (IntuloxP/loxP; Krt14-Cre) allowed us to overcome embryonic lethal phenotypes and determine the function of Intu throughout the morphogenetic processes of the skin and the hair follicles. To our surprise, targeting Krt14-expressing cells not only resulted in hair follicle development abnormalities, but also growth retardation and perinatal death. Although skin-related abnormalities have unlikely contributed to lethality, we are not yet able to determine the exact cause. Because Krt14-Cre also targets cells of other epithelial tissues, such as the mucus membrane of upper respiratory track, esophagus, and the forestomach, which contain primary (and motile) cilia, the lethal phenotypes suggest important function of Intu in the development and function of these epithelial tissue types.

In summary, this study identified a critical role of a PCP effector gene, Intu, in the differentiation of epidermal keratinocytes after their commitment to a follicular fate, suggesting a potential mechanism through which PCP signaling executes its function in a cell fate- and contextdependent manner. Our findings demonstrate that the epidermis and hair follicles are excellent model systems in dissecting the PCP signaling pathway in mammals.

\footnotetext{
Materials and Methods

Animals and tissue preparation. The Intu ${ }^{-/-}$and IntuloxP/loxP mice were reported previously. ${ }^{18}$ Wild-type or heterozygous $\left(I n t u^{+/-}\right)$littermates were used as controls. $\mathrm{Tg}(\mathrm{KRT} 14-\mathrm{cre}) 1 \mathrm{Amc} / \mathrm{J}$ mice were obtained from the Jackson Laboratory (Bar Harbor, ME, USA). ${ }^{51}$ Intu ${ }^{\text {loxP/loxP }} ;$ Krt14-Cre mice were obtained by crossing IntuloxP/loxP with IntuloxP;Tg(KRT14-cre)1 Amc/J mice. Intu ${ }^{+/ \text {loxP }} ; \mathrm{Krt14}-\mathrm{Cre}$ or IntuloxPlloxP mice were used as controls.

Embryos or dorsal skins were fixed overnight in 10\% buffered formalin immediately after dissection and processed for routine histology. All work related to mice was performed in accordance with the guidelines developed by the institutional animal use and care committee of the University of Colorado Anschutz Medical Campus and the Pennsylvania State University.
}

RNA preparation and qRT-PCR. Total RNA was extracted using RNeasy Mini Kits (Qiagen, Valencia, CA, USA) according to the manufacturer's instructions. High-Capacity cDNA Archive Kit (Applied Biosystems, Carlsbad, CA, USA) was used for CDNA synthesis. Relative gene expression levels were determined by qRT-PCR. Assays-on-Demand Taqman probes for Intu (ABI, Mm01284305_m1), Ptch (Mm01306905_m1), Gli1 (Mm00494645_m1), Gli3 (Mm00492333_m1), and glyceraldehyde 3-phosphate dehydrogenase (GAPDH; 4352339E) were obtained from Applied Biosystems; Krt14 (Mm.PT.49a.17599324.gs), Krt10 (Mm.PT.49a.16714815.gs), Flg (Mm.PT. 49a.21901092.g), Lor (Mm.PT.49a.12882060), Notch3 (Mm.PT.49a.5191442.g), and Psen2 (Mm.PT.49a.21655208) were obtained from Integrated DNA Technologies (Coralville, IA, USA). Results were analyzed using a standard curve method. The expression of each target gene was normalized to that of GAPDH to obtain a relative expression level. 
Immunofluorescence labeling and microscopy. Paraffin-embedded tissue sections were deparaffinized, rehydrated, and boiled in $10 \mathrm{mM}$ sodium citrate $(\mathrm{pH}$ 6.0) for $10 \mathrm{~min}$ as an antigen retrieval step. For labeling primary cilia, $0.1 \mathrm{mM}$ ethylenediaminetetraacetic acid (EDTA; pH 8.0) was used in place of sodium citrate. Sections were incubated with primary antibodies diluted in $5 \%$ goat serum at $4{ }^{\circ} \mathrm{C}$ overnight. The following antibodies and dilutions were used: $\mathrm{p} 63$ (1: 100, Santa Cruz Biotechnology, Santa Cruz, CA, USA), Krt71 (1:100, K6irs1, a gift from Dr. Yutaka Shimomura), Arl13B (ADP-ribosylation factor-like 13B; 1: 1500, a gift from Tamara Caspary), $\gamma$-tubulin (Tubg; 1: 1000, DM1A; Sigma, St. Louis, MO, USA), Vangl1 (1:500, HPA025235; Sigma), p75 NTR (1:200, G3231; Promega, Madison, WI, USA), Lef1 (1:100, C12A5; Cell Signaling, Danvers, MA, USA), bromodeoxyuridine (BrdU; 1:20, A21304; Invitrogen, Grand Island, NY USA), Krt75 (Chen et al.), ${ }^{57}$ and AE13 (1:100, ab16113, Abcam, Cambridge, MA, USA). The Krt14, Krt10, and Lor antibodies were generated by Roop et al. ${ }^{58}$ The following day, sections were incubated in secondary antibodies Alexa-conjugated fluorochrome 594 or 488 anti-IgG to the corresponding host species of the primary antibodies (Molecular Probes, Eugene, OR, USA). Nikon Eclipse 90i microscope (Nikon Melville, NY, USA) was used for photography.

BrdU incorporation and TUNEL (terminal deoxynucleotidyl transferase-mediated deoxyuridine triphosphate nick-end labeling) analyses. To determine the proliferative index, mice were injected i.p. with $10 \mu \mathrm{l} / \mathrm{g}$ of BrdU labeling reagent (Zymed, Life Technologies, Grand Island, $\mathrm{NY}, \mathrm{USA}$ ). Tissues were collected $2 \mathrm{~h}$ later and embedded tissues were stained with an Alexa Fluor dye-conjugated anti-BrdU antibody (Invitrogen). DeadEnd Fluorometric TUNEL system (Promega) was used according to the manufacturer's instruction manual to measure nuclear DNA fragmentation.

Skin separation and transplantation. Skins were dissected from E18.5 embryos and floated on $1.5 \mathrm{U} / \mathrm{ml}$ dispase II (Roche Applied Science, Indianapolis, IN, USA) for $1 \mathrm{~h}$ before separating the epidermis and dermis by peeling. Keratinocytes and dermal fibroblasts were harvest as described by Lichti et al..$^{59}$

Full-thickness E18.5 embryonic skins were transplanted to Foxn ${ }^{-1-}$ nude mice as described in our previous study. ${ }^{29}$ At 5 weeks after transplantation, skin grafts were harvested for analysis.

Microscopy and image processing. Photographs were taken with a Nikon Eclipse 90i microscope in conjunction with the NIS-Elements AR 3.0 imaging software (Nikon). Images were processed with Adobe Photoshop CS5 Extended and assembled with Adobe lllustrator CS3 (Adobe, San Jose, CA, USA).

Transmission electron microscopy. Small pieces of back skin of E18.5 embryos were fixed in $4 \%$ paraformaldehyde, $2.5 \%$ gluteraldehyde in $0.1 \mathrm{M}$ sodium cacodylate $(\mathrm{pH} 7.4)$ at $4^{\circ} \mathrm{C}$ overnight. After several washes in $0.1 \mathrm{M}$ cacodylate buffer ( $\mathrm{pH} 7.4)$, samples were postfixed in $1 \%$ osmium tetroxide in $0.1 \mathrm{M}$ sodium cacodylate buffer for $1 \mathrm{~h}$ at room temperature. Samples were then dehydrated through a graded ethanol series, and then embedded in a mixture of epon-araldite resin for overnight at $60^{\circ} \mathrm{C}$. Finally, thin sections were cut and poststained with lead citrate and uranyl acetate before been examined on an FEI Technai G2 electron microscope (Hillsboro, OR, USA).

Whole-mount dye penetration assay. Epidermal barrier was examined by a whole-mount dye penetration assay. E18.5 control and mutant embryos were dissected out carefully, washed in phosphate-buffered saline (PBS), and dehydrated by incubating in serial concentrations of methanol $(25,50,75$, and $100 \%$ methanol in PBS) for 1 min per dilution. The embryos were then rehydrated by reversing the incubations back to $100 \%$ PBS for 1 min per incubation. Embryos were then stained for $1 \mathrm{~min}$ in $0.0125 \%$ toluidine blue 0 in PBS (Fisher Scientific, Pittsburgh, PA, USA) before embryos were washed in PBS to destain, and were then photographed.

Statistical analysis. Each experiment was performed minimum three times unless otherwise stated. Representative images were used in the article. Student's $t$-test was used to calculate statistically significant differences. $P<0.05$ was considered statistically significant.

\section{Conflict of Interest}

The authors declare no conflict of interest.
Acknowledgements. We thank Dr. Dennis R Roop for helpful discussion, Christy Ou for assistance in statistical analyses and generating figures, Dorothy Dill (UCD EM Center) for assistance in transmission electron microscopy, Dr. Tamara Caspary for providing the Arl13b antibody, and Dr. Yutaka Shimomura for the KRT71 antibody. This study was supported in part by an International Union Against Cancer (UICC) and American Cancer Society Beginning Investigators Fellowship grant to LL; a Young Investigator Development Award from the PKD foundation to AL; a P\&F grant and core services from the UCD Skin Disease Research Center (SDRC), and a research grant from NIH/NIAMS (AR061485) to JC.

\section{Author contributions}

DD, LL, DJC, and JC designed and performed experiments; DD, EG, AH, and JC collected and analyzed data; LL, HZ, EG, JC, and AL designed and performed experiments with the germline mutants of $I n t u ; \mathrm{DD}, \mathrm{AL}$, and JC wrote the manuscript.

1. Song $\mathrm{H}, \mathrm{Hu}$ J, Chen W, Elliott G, Andre P, Gao B et al. Planar cell polarity breaks bilateral symmetry by controlling ciliary positioning. Nature 2010; 466: 378-382

2. Borovina A, Superina S, Voskas D, Ciruna B. Vangl2 directs the posterior tilting and asymmetric localization of motile primary cilia. Nat Cell Biol 2010; 12: 407-412.

3. Tissir F, Qu Y, Montcouquiol M, Zhou L, Komatsu K, Shi D et al. Lack of cadherins Celsr2 and Celsr3 impairs ependymal ciliogenesis, leading to fatal hydrocephalus. Nat Neurosci 2010; 13: 700-707.

4. Hashimoto M, Shinohara K, Wang J, Ikeuchi S, Yoshiba S, Meno C et al. Planar polarization of node cells determines the rotational axis of node cilia. Nat Cell Biol2010; 12: 170-176

5. Antic D, Stubbs JL, Suyama K, Kintner C, Scott MP, Axelrod JD. Planar cell polarity enables posterior localization of nodal cilia and left-right axis determination during mouse and Xenopus embryogenesis. PLoS One 2010; 5: e8999.

6. Wallingford JB. Planar cell polarity, ciliogenesis and neural tube defects. Hum Mol Genet 2006; 15(Spec No 2): R227-R234.

7. Wallingford JB, Mitchell B. Strange as it may seem: the many links between Wnt signaling, planar cell polarity, and cilia. Genes Dev 2011; 25: 201-213.

8. Rida PC, Chen P. Line up and listen: planar cell polarity regulation in the mammalian inner ear. Semin Cell Dev Biol 2009; 20: 978-985.

9. Bayly R, Axelrod JD. Pointing in the right direction: new developments in the field of planar cell polarity. Nat Rev Genet 2011; 12: 385-391.

10. Simons M, Mlodzik M. Planar cell polarity signaling: from fly development to human disease. Annu Rev Genet 2008; 42: 517-540.

11. Hamblet NS, Lijam N, Ruiz-Lozano P, Wang J, Yang Y, Luo Z et al. Dishevelled 2 is essential for cardiac outflow tract development, somite segmentation and neural tube closure. Development 2002; 129: 5827-5838.

12. Etheridge SL, Ray S, Li S, Hamblet NS, Lijam N, Tsang M et al. Murine dishevelled 3 functions in redundant pathways with dishevelled 1 and 2 in normal cardiac outflow tract, cochlea, and neural tube development. PLoS Genet 2008; 4: e1000259.

13. Kibar Z, Vogan KJ, Groulx N, Justice MJ, Underhill DA, Gros P. Ltap a mammalian homolog of Drosophila Strabismus/Van Gogh, is altered in the mouse neural tube mutant Loop-tail. Nat Genet 2001; 28: 251-255.

14. Murdoch JN, Doudney K, Paternotte C, Copp AJ, Stanier P. Severe neural tube defects in the loop-tail mouse result from mutation of Lpp1, a novel gene involved in floor plate specification. Hum Mol Genet 2001; 10: 2593-2601.

15. Heydeck W, Zeng H, Liu A. Planar cell polarity effector gene Fuzzy regulates cilia formation and Hedgehog signal transduction in mouse. Dev Dyn 2009; 238: 3035-3042.

16. Gray RS, Abitua PB, Wlodarczyk BJ, Szabo-Rogers HL, Blanchard O, Lee I et al. The planar cell polarity effector Fuz is essential for targeted membrane trafficking, ciliogenesis and mouse embryonic development. Nat Cell Biol 2009; 11: 1225-1232.

17. Kim SK, Shindo A, Park TJ, Oh EC, Ghosh S, Gray RS et al. Planar cell polarity acts through septins to control collective cell movement and ciliogenesis. Science 2010; 329 : 1337-1340.

18. Zeng $\mathrm{H}$, Hoover AN, Liu A. PCP effector gene Inturned is an important regulator of cilia formation and embryonic development in mammals. Dev Biol 2010; 339: 418-428.

19. Blanpain C, Fuchs E. Epidermal homeostasis: a balancing act of stem cells in the skin. Nat Rev Mol Cell Biol 2009; 10: 207-217.

20. Devenport D, Oristian D, Heller E, Fuchs E. Mitotic internalization of planar cell polarity proteins preserves tissue polarity. Nat Cell Biol 2011; 13: 893-902.

21. Lefort K, Mandinova A, Ostano P, Kolev V, Calpini V, Kolfschoten I et al. Notch1 is a p53 target gene involved in human keratinocyte tumor suppression through negative regulation of ROCK $1 / 2$ and MRCKalpha kinases. Genes Dev 2007; 21: 562-577.

22. Kwei KA, Finch JS, Ranger-Moore J, Bowden GT. The role of Rac1 in maintaining malignant phenotype of mouse skin tumor cells. Cancer Lett 2006; 231: 326-338.

23. Liu AX, Rane N, Liu JP, Prendergast GC. RhoB is dispensable for mouse development, but it modifies susceptibility to tumor formation as well as cell adhesion and growth factor signaling in transformed cells. Mol Cell Biol 2001; 21: 6906-6912.

24. Caddy J, Wilanowski T, Darido C, Dworkin S, Ting SB, Zhao Q et al. Epidermal wound repair is regulated by the planar cell polarity signaling pathway. Dev Cell 2010; 19: 138-147. 
25. Guo N, Hawkins C, Nathans J. Frizzled6 controls hair patterning in mice. Proc Natl Acad Sci USA 2004; 101: 9277-9281.

26. Devenport D, Fuchs E. Planar polarization in embryonic epidermis orchestrates global asymmetric morphogenesis of hair follicles. Nat Cell Biol 2008; 10: 1257-1268.

27. Ravni A, Qu Y, Goffinet AM, Tissir F. Planar cell polarity cadherin Celsr1 regulates skin hair patterning in the mouse. J Invest Dermatol 2009; 129: 2507-2509.

28. Simons M, Gloy J, Ganner A, Bullerkotte A, Bashkurov M, Kronig C et al. Inversin, the gene product mutated in nephronophthisis type II, functions as a molecular switch between Wnt signaling pathways. Nat Genet 2005; 37: 537-543.

29. Dai D, Zhu H, Wlodarczyk B, Zhang L, Li L, Li AG et al. Fuz controls the morphogenesis and differentiation of hair follicles through the formation of primary cilia. $J$ Invest Dermatol 2011; 131: 302-310.

30. Chrostek A, Wu X, Quondamatteo F, Hu R, Sanecka A, Niemann C et al. Rac1 is crucial for hair follicle integrity but is not essential for maintenance of the epidermis. Mol Cell Biol 2006; 26: 6957-6970.

31. Wu X, Quondamatteo F, Lefever T, Czuchra A, Meyer H, Chrostek A et al. Cdc42 controls progenitor cell differentiation and beta-catenin turnover in skin. Genes Dev 2006; 20: 571-585.

32. Botchkarev VA, Paus R. Molecular biology of hair morphogenesis: development and cycling. J Exp Zool B Mol Dev Evol 2003; 298: 164-180.

33. Schmidt-Ullrich R, Paus R. Molecular principles of hair follicle induction and morphogenesis. Bioessays 2005; 27: 247-261.

34. Andl T, Reddy ST, Gaddapara T, Millar SE. WNT signals are required for the initiation of hair follicle development. Dev Cell 2002; 2: 643-653.

35. Chan EF, Gat U, McNiff JM, Fuchs E. A common human skin tumour is caused by activating mutations in beta-catenin. Nat Genet 1999; 21: 410-413.

36. Gat U, DasGupta R, Degenstein L, Fuchs E. De novo hair follicle morphogenesis and hair tumors in mice expressing a truncated beta-catenin in skin. Cell 1998; 95: 605-614.

37. Zhang Y, Andl T, Yang SH, Teta M, Liu F, Seykora JT et al. Activation of beta-catenin signaling programs embryonic epidermis to hair follicle fate. Development 2008; 135 : 2161-2172.

38. St-Jacques B, Dassule HR, Karavanova I, Botchkarev VA, Li J, Danielian PS et al. Sonic hedgehog signaling is essential for hair development. Curr Biol 1998; 8: 1058-1068.

39. Mill P, Mo R, Fu H, Grachtchouk M, Kim PC, Dlugosz AA et al. Sonic hedgehog-dependent activation of Gli2 is essential for embryonic hair follicle development. Genes Dev 2003; 17 : 282-294.

40. Chiang C, Swan RZ, Grachtchouk M, Bolinger M, Litingtung Y, Robertson EK et al. Essential role for Sonic hedgehog during hair follicle morphogenesis. Dev Biol 1999; 205: $1-9$.

41. Epstein EH. Basal cell carcinomas: attack of the hedgehog. Nat Rev Cancer 2008; 8 : 743-754.

42. Plikus MV, Baker RE, Chen CC, Fare C, de la Cruz D, Andl T et al. Self-organizing and stochastic behaviors during the regeneration of hair stem cells. Science 2011; 332 : 586-589.
43. Plikus MV, Mayer JA, de la Cruz D, Baker RE, Maini PK, Maxson R et al. Cyclic dermal BMP signalling regulates stem cell activation during hair regeneration. Nature 2008; 451 : 340-344.

44. Ito M, Yang Z, Andl T, Cui C, Kim N, Millar SE et al. Wnt-dependent de novo hair follicle regeneration in adult mouse skin after wounding. Nature 2007; 447: 316-320.

45. Singla V, Reiter JF. The primary cilium as the cell's antenna: signaling at a sensory organelle. Science 2006; 313: 629-633.

46. Goetz SC, Anderson KV. The primary cilium: a signalling centre during vertebrate development. Nat Rev Genet 2010; 11: 331-344.

47. Lehman JM, Laag E, Michaud EJ, Yoder BK. An essential role for dermal primary cilia in hair follicle morphogenesis. J Invest Dermatol 2009; 129: 438-448.

48. Ezratty EJ, Stokes N, Chai S, Shah AS, Williams SE, Fuchs E. A role for the primary cilium in Notch signaling and epidermal differentiation during skin development. Cell 2011; 145: 1129-1141.

49. Schneider L, Cammer M, Lehman J, Nielsen SK, Guerra CF, Veland IR et al. Directional cell migration and chemotaxis in wound healing response to PDGF-AA are coordinated by the primary cilium in fibroblasts. Cell Physiol Biochem 2010; 25: 279-292.

50. Wong SY, Seol AD, So PL, Ermilov AN, Bichakjian CK, Epstein EH Jr. et al. Primary cilia can both mediate and suppress Hedgehog pathway-dependent tumorigenesis. Nat Med 2009; 15: 1055-1061.

51. Dassule HR, Lewis P, Bei M, Maas R, McMahon AP. Sonic hedgehog regulates growth and morphogenesis of the tooth. Development 2000; 127: 4775-4785.

52. Paus R, Muller-Rover S, Van Der Veen C, Maurer M, Eichmuller S, Ling G et al. A comprehensive guide for the recognition and classification of distinct stages of hair follicle morphogenesis. J Invest Dermatol 1999; 113: 523-532.

53. Park TJ, Haigo SL, Wallingford JB. Ciliogenesis defects in embryos lacking inturned or fuzzy function are associated with failure of planar cell polarity and Hedgehog signaling. Nat Genet 2006; 38: 303-311.

54. Heydeck W, Liu A. PCP effector proteins inturned and fuzzy play nonredundant roles in the patterning but not convergent extension of mammalian neural tube. Dev Dyn 2011; 240: 1938-1948.

55. Croyle MJ, Lehman JM, O'Connor AK, Wong SY, Malarkey EB, Iribarne D et al. Role of epidermal primary cilia in the homeostasis of skin and hair follicles. Development 2011; 138: $1675-1685$.

56. Schneider MR, Schmidt-Ullich R, Paus R. The hair follicle as a dynamic miniorgan. Curr Biol 2009; 19: R132-R142.

57. Chen J, Jaeger K, Den Z, Koch PJ, Sundberg JP, Roop DR. Mice expressing a mutant Krt75 (K6hf) allele develop hair and nail defects resembling pachyonychia congenita. $J$ Invest Dermatol 2008; 128: 270-279.

58. Roop DR, Huitfeldt H, Kilkenny A, Yuspa SH. Regulated expression of differentiationassociated keratins in cultured epidermal cells detected by monospecific antibodies to unique peptides of mouse epidermal keratins. Differentiation 1987; 35: 143-150.

59. Lichti U, Anders J, Yuspa SH. Isolation and short-term culture of primary keratinocytes, hair follicle populations and dermal cells from newborn mice and keratinocytes from adult mice for in vitro analysis and for grafting to immunodeficient mice. Nat Protoc 2008; 3: 799-810. 